\title{
Chronic Nitric Oxide Deficiency and Progression of Kidney Disease after Renal Mass Reduction in the C57BI6 Mouse
}

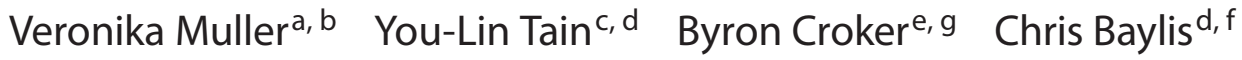 \\ a Department of Pulmonology, Semmelweis University, Budapest, Hungary; ${ }^{b}$ Department of Physiology, West \\ Virginia University, Morgantown, W. Va., USA; 'Department of Pediatrics, Chang Gung Memorial Hospital, \\ Kaohsiung Medical Center, Chang Gung University, Kaohsiung, Taiwan, ROC; Departments of d Physiology and \\ Functional Genomics, ePathology, Immunology and Laboratory Medicine, and ${ }^{\mathrm{f}}$ Medicine, University of Florida, \\ and 9Pathology and Laboratory Medicine Service, North Florida/South Georgia Veterans Health System, \\ Gainesville, Fla., USA
}

\section{Key Words}

Nitric oxide synthase inhibition - Endothelial NOS knockout • Albuminuria $\cdot$ Creatinine clearance $\cdot$ Renal pathology

\begin{abstract}
Background/Aims: The C57BI6 mouse is resistant to chronic kidney disease (CKD) induced by reduction of renal mass (RRM). Nitric oxide (NO) deficiency exacerbates CKD progression so this study investigated whether combination of RRM and NO deficiency would render the C57BI6 mouse vulnerable to CKD. Methods: We used wild-type (WT) mice with RRM, chronic NO synthase (NOS) inhibition and a combination. Also, endothelial NOS (eNOS) knockout (KO) C57Bl6 mice were studied with and without RRM. Primary endpoints were albuminuria and structural damage. Results: Both nonselective (+L-NAME) and neuronal NOS 'selective' (+7NI) NOS inhibition greatly exacerbated the albuminuria and structural damage seen with RRM in the WT mice; NOS inhibition alone had little effect. The eNOS KO mice showed marked structural damage and significant albuminuria in the shams and RRM produced minimal exacerbation of structural damage although the albuminuria was massively amplified. Conclusion: These studies demonstrate that the C57BI6 mouse is rendered vulnerable to RRM-induced CKD when concom-
\end{abstract}

itant NO deficiency is produced. This observation supports previous work in CKD-resistant rats and suggests that NO deficiency is required for progression of CKD.

Copyright $\odot 2010$ S. Karger AG, Basel

\section{Introduction}

There are marked species and strain differences in the susceptibility to chronic kidney disease (CKD), for example the Wistar-Furth (WF) rat and the C57Bl6 mouse are resistant to progression of renal ablation-induced injury [1-3]. In the WF rat the protection from injury is related to relative preservation of the renal and overall nitric oxide (NO) systems $[1,4]$. In vulnerable animals (i.e. those susceptible to progression of CKD) NO deficiency develops due to endothelial dysfunction due to increased endogenous NOS inhibitor ADMA and oxidative stress, possible substrate (L-arginine) deficiency at the active site of the NOS and loss of renal NO synthase [1]. Our recent observations in the rat suggest that the renal neuronal NO synthase (nNOS) response to kidney damage may be an important determinant of the susceptibility to CKD progression $[1,5]$.

\section{KARGER}

(C) 2010 S. Karger AG, Basel

Fax +4161306 1234 E-Mail karger@karger.ch www.karger.com www.karger.com/ajn
Chris Baylis, $\mathrm{PhD}$

Department of Physiology and Functional Genomics

University of Florida, 1600 SW Archer Road

PO Box 100274, Gainesville, FL 36210 (USA)

Tel. +1 352392 7869, Fax +1 352392 7935, E-Mail baylisc@ ufl.edu 
Table 1. 24-Hour urinary albumin excretion (mg/day)

\begin{tabular}{lcccc}
\hline Group & Week 0 & Week 4 & Week 8 & Week 11 \\
\hline 1 WT sham $(\mathrm{n}=4)$ & $22 \pm 3$ & $21 \pm 6$ & $20 \pm 3$ & $26 \pm 5$ \\
2 WT L-NAME $(\mathrm{n}=4)$ & $18 \pm 6$ & $15 \pm 5$ & $14 \pm 6$ & $28 \pm 7$ \\
3 WT 7NI (n=6) & $24 \pm 3$ & $33 \pm 6$ & $42 \pm 6^{\mathrm{a}}$ & $43 \pm 4^{\mathrm{a}}$ \\
4 WT CN $(\mathrm{n}=6)$ & $25 \pm 1$ & $30 \pm 1$ & $45 \pm 8^{\mathrm{a}}$ & $68 \pm 14^{\mathrm{a}}$ \\
5 WT CN/L-NAME $(\mathrm{n}=6)$ & $22 \pm 2$ & $34 \pm 6$ & $106 \pm 35^{\mathrm{a}}$ & $383 \pm 194^{\mathrm{a}}$ \\
6 WT CN/7NI $(\mathrm{n}=5)$ & $35 \pm 2$ & $143 \pm 29^{\mathrm{a}}$ & $162 \pm 30^{\mathrm{a}}$ & $248 \pm 67^{\mathrm{a}}$ \\
7 eNOS KO sham $(\mathrm{n}=4)$ & $62 \pm 10$ & $55 \pm 6$ & $133 \pm 30^{\mathrm{a}}$ & $162 \pm 17^{\mathrm{a}}$ \\
8 eNOS KO CN $(\mathrm{n}=5)$ & $124 \pm 29$ & $648 \pm 169^{\mathrm{a}}$ & $964 \pm 67^{\mathrm{a}}$ & $1,170 \pm 44^{\mathrm{a}}$ \\
\hline
\end{tabular}

${ }^{\mathrm{a}} \mathrm{p}<0.05$ denotes difference from the WT sham value at a given observation week.
In this study we investigate the importance of the NOS system in the C57Bl6 mouse with a renal ablation model using cauterization of the left kidney cortex and removal of the right kidney 1 week later $(\mathrm{CN})$; mice were studied after 11 weeks. To manipulate NOS, we used systemic nonselective NOS inhibition (with L-NAME), 'selective' nNOS inhibition (with 7NI) and the endothelial (e)NOS knockout mice (on the C57Bl6 background). The primary endpoints were albuminuria and histologic evidence of renal structural injury.

\section{Methods}

Studies were conducted on 31 C57/BL6 male mice and 9 NOS3 knockouts on the C57BL6 background (Jackson Labs, Bar Harbor, Me., USA). Either sham or CN surgery was performed at $\sim 16$ weeks of age, as follows: $10 \mathrm{~min}$ after IP atropine $(0.2 \mathrm{mg} / \mathrm{kg})$ mice were anesthetized with $35 \mathrm{mg} / \mathrm{kg}$ b.w. pentobarbital sodium (Sigma, St. Louis, Mo., USA) and $17 \mathrm{mg} / \mathrm{kg}$ b.w. IP methohexital sodium (brevital sodium, Eli Lilly \& Co., Indianapolis, Ind., USA). $\mathrm{CN}$ was performed by cauterization of the left kidney cortex and right kidney nephrectomy 1 week later, as described previously [6]. Controls were subjected to two sham operations.

One day after the second surgery the $\mathrm{CN}$ and sham operated mice were divided into the following treatment groups: group 1 $(n=4)$ were sham controls, group $2(n=4)$ were sham controls given chronic nonselective NOS inhibition $(0.2 \mathrm{~g} / \mathrm{l} \mathrm{L} \omega$-nitro-Larginine methyl ester (L-NAME; Sigma), dissolved in drinking water and changed every other day), group $3(n=6)$ were sham controls given chronic selective neuronal NOS inhibitor, 7-nitroindazole (7NI; $0.1 \mathrm{~g} / \mathrm{l}$ in drinking water), group $4(\mathrm{n}=6)$ were $\mathrm{CN}$, group $5(n=6)$ were $C N+$ chronic L-NAME, and group $6(n=5)$ were $\mathrm{CN}$ given chronic 7NI. Doses of L-NAME and 7NI were selected based on the study by Kurihara et al. [7]. Groups 1-6 were wild-type (WT) C57Bl6, groups 7 and 8 were eNOS knockouts, group $7(n=4)$ were sham controls, and group $8(n=5)$ were $C N$.

Metabolic cage collections of urine were made before surgery and at 4, 8 and 11 weeks for measurement of albumin and creatinine. After the week 11 metabolic cage urine collection, mice were anesthetized (as above) and a 27-gauge needle connected to a pres- sure transducer was inserted into the abdominal aorta for blood pressure (BP) measurement. An aortic blood sample was obtained for creatinine and NOx (nitrite + nitrate) measurement and the left kidney was removed, cut in half and fixed in $10 \%$ buffered formalin for histology.

Urine albumin was measured by ELISA (Bethyl Laboratories, Inc., Montgomery, Tex., USA). Plasma NOx was measured using the NOx fluorimetric assay (Cayman, Ann Arbor, Mich., USA). Plasma and urine creatinine was measured by HPLC, described by us previously [4].

Kidney sections were fixed in $10 \%$ buffered formalin, embedded in paraffin wax, sectioned in $5-\mu \mathrm{m}$ slices and stained with PAS. Kidney sections were evaluated on a blinded basis (by B.C.) for determination of global glomerulosclerosis (GS), segmental GS, mesangial matrix (MM) expansion, glomerular ischemia/ atrophy, tubular atrophy, tubular hypertrophy, interstitial fibrosis, and interstitial inflammation. Each element is scaled $0-5$ as follows: none (0), $1-10 \%$ (1), $11-25 \%$ (2), $26-50 \%$ (3), $51-75 \%$ (4), and 76-100 (5) as described previously [8].

Results are presented as mean \pm SEM. Parametric data was analyzed by t test and one-way ANOVA. Nonparametric data was tested using the Kruskal-Wallis one-way analysis of ranks. $\mathrm{p}<$ 0.05 was considered statistically significant.

\section{Results}

As shown in table 1, urinary albumin excretion (UalbV) was low and similar in groups 1-6 (all WT) at baseline (week 0). Sham rats and shams given chronic nonselective NOS inhibition with oral L-NAME showed no change in UalbV over the 11-week follow-up period. Shams given 'selective' nNOS inhibition with 7NI developed mild albuminuria which was also seen in the $\mathrm{CN}$ mice. The combination of L-NAME and CN (group 5) produced significant albuminuria at week 4 which was marked at weeks 8 and 11 post-surgery. The combination of 7NI with $\mathrm{CN}$ also led to marked albuminuria from week 4 onwards. In contrast to the WT mice, the eNOS 
Table 2. Histological analysis on renal cortex



All numbers are given in table $1 .{ }^{\mathrm{a}} \mathrm{p}<0.05$ vs. CN/7NI. ${ }^{\mathrm{b}} \mathrm{p}<0.05$ vs. WT sham.

knockouts had substantial baseline albuminuria which increased $\sim 2.5 \times$ over the 11-week observation period in the shams. The addition of $\mathrm{CN}$ in the eNOS knockouts led to massive albuminuria following $\mathrm{CN}$ with $\sim 10 \times$ increase by 11 weeks post-surgery (table 1 ).

Table 2 summarizes the histological evaluation on renal cortex, made at 11 weeks after $\mathrm{CN}$ or sham surgery. The sham WT kidneys showed no glomerular damage and minimal tubular atrophy. Chronic L-NAME had no impact on any variable except for mild glomerular ischemia/atrophy; the tubulointerstitium was similar to shams. Shams given chronic 7NI ('selective' nNOS inhibitor) showed a striking increase in $M M$ expansion, which affected most $(>80 \%)$ glomeruli, as well as the appearance of mild segmental GS, interstitial fibrosis and inflammation. CN in WT led to global GS in about $5 \%$ of glomeruli and mild MM expansion in $\sim 10 \%$ of glomeruli as well as glomerular ischemia/atrophy in $\sim 50 \%$ of glomeruli. There was also mild tubular atrophy and hypertrophy as well as interstitial fibrosis and inflammation affecting $\sim 10 \%$ of the tubulointerstitium. When $\mathrm{CN}$ was combined with either L-NAME or 7NI there was a numerically, but not statistically significant increase in both global and segmental GS compared to the untreated WT with CN. There was substantial MM expansion in $\sim 50 \%$ of glomeruli with the combination of $\mathrm{CN}+\mathrm{L}-$ NAME and significantly greater MM expansion in the $\mathrm{CN}+7 \mathrm{NI}$ mice. The tubulointerstitial changes were relatively mild and similar in groups 5 and 6 . The sham eNOS KO exhibited $\sim 10 \%$ global and 5\% segmental GS with MM expansion in the majority of glomeruli, 5\% glomerular ischemia/atrophy and mild tubulointerstitial injury. Remarkably, the addition of CN (group 8) did not significantly worsen any of the histological findings (ta- ble 2) although the albuminuria was massively increased, see above. Representative sections are shown in figure 1.

We obtained a terminal BP in the 6 WT C57Bl6 groups and as shown in table 3 there were no differences in mean BP between any of the six groups. Unfortunately, we did not obtain any BP measurements in the eNOS KOs.

We assessed NO production from plasma NOx (factored for creatinine to control for differences in renal clearance). In WT shams PNOx/cr was not different to the WT L-NAME group, WT 7NI or WT CN (table 3). However, with the combination of $\mathrm{CN}+\mathrm{L}-\mathrm{NAME}$ and $\mathrm{CN}+7 \mathrm{NI}$ the $\mathrm{PNOx} / \mathrm{cr}$ was significantly lower than the WT sham value (table 3). In eNOS KO shams, PNOx/cr was markedly lower than in WT shams and PNOx/cr in eNOS KO $\mathrm{CN}$ rats was also much reduced versus $\mathrm{WT}$ shams (table 3). The 24-hour creatinine clearances (Ccr) are also given in table 3 . As expected, $\mathrm{CN}$ always reduced Ccr compared to sham control. The Ccr was not different between the WT sham and the eNOS KO; however, unexpectedly, 11 weeks of both L-NAME and 7NI led to significant increases in Ccr versus WT shams, perhaps reflecting an increase in glomerular BP and filtration fraction.

\section{Discussion}

The main novel findings in this study were that the combination of $\mathrm{CN}$ and nonselective (+L-NAME) or nNOS 'selective' (+7N1) inhibition render the renal injury-resistant C57Bl6 mouse susceptible to kidney damage. As reported by others, the eNOS KO mouse on the C57Bl6 background exhibits significant structural injury and albuminuria and while $\mathrm{CN}$ did not worsen the structural damage it caused exacerbation of the albuminuria. 
Fig. 1. a, b Normal glomeruli and tubules in the sham WT and the L-NAME-treated WT. c Two glomeruli with significant mesangial matrix expansion (MM expansion) in the 7NI-treated WT. d An area of focal tubulointerstitial fibrosis and inflammation in the WT mice subjected to cautery/nephrectomy (CN). e Significant glomerular sclerosis in WT mouse with $\mathrm{CN}$ and given L-NAME. f Global MM expansion in the upper glomerulus and an obsolescent glomerulus, below. g Obsolescent glomerulus in the eNOS knockout sham. h An obsolescent glomerulus and a normal glomerulus with MM expansion in the eNOS knockout with CN.
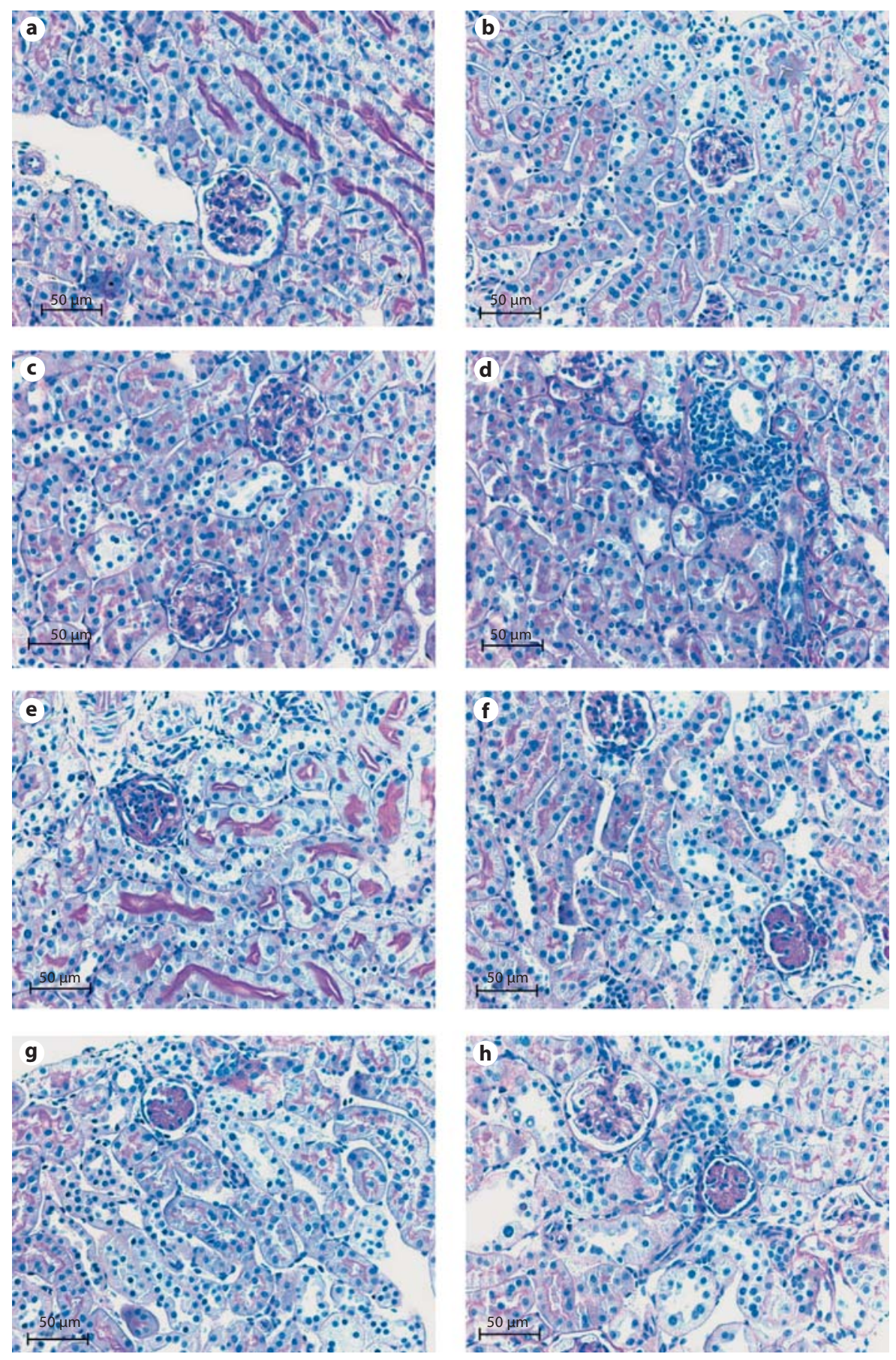

Several lines of evidence suggest that progression of CKD involves development of NO deficiency [1]. The WF rat is highly resistant to progression of CKD irrespective of the initiating cause $[4,9,10]$ and has a robust and wellpreserved NOS system. The WF is also resistant to CKD and hypertension induced by chronic, high-dose NOS inhibition with L-NAME [11]. However, when we combined severe renal mass reduction with low-dose chronic NOS inhibition the WF developed rapidly progressing CKD [4]. This suggests that NO deficiency and a second 'hit' must be present for progression of CKD. In the present study we demonstrate a similar relationship between NO deficiency and progression of renal mass reduction-induced CKD in the injury-resistant C57Bl6 mouse. Our observations 
with the $\mathrm{CN}$ model confirm earlier studies which used different renal mass reduction models and showed that the C57Bl6 is resistant to this form of CKD $[2,3]$. Here we report that chronic nonselective NOS inhibition with LNAME had no impact on albumin excretion, renal structure/fibrosis or BP when given for 11 weeks in the present study. In contrast, in a transgenic mouse of undisclosed background strain, administration of 14 weeks of chronic L-NAME (same dose as used by us) led to marked glomerular sclerosis and hypertension [12], thus the C57Bl6 may also be resistant to systemic NOS inhibition.

Expansion of the MM is a precursor of glomerular sclerosis and while chronic NOS inhibition with L-NAME alone caused no MM expansion, the nNOS 'selective' NOS inhibitor 7NI given alone led to widespread MM expansion (table 2). This may be because at the dose administered, 7NI was a more potent NOS inhibitor than $\mathrm{L}-\mathrm{NAME}$, supported by the lower value of $\mathrm{PNOx} / \mathrm{cr}$ seen with $7 \mathrm{NI}$ versus L-NAME $(1.53 \pm 0.31$ vs. $5.2 \pm 0.45$, $\mathrm{p}<0.05)$. Again, the combination of chronic NOS inhibition (with 7NI) and $\mathrm{CN}$ led to increased renal structural damage and albuminuria. It should be noted that although no differences in BP were seen with either method of NOS inhibition, the BP measurements were obtained under general anesthesia which may have lowered all values, although we anticipate that differences between groups would still be detectable.

The reason that we gave the $7 \mathrm{NI}$ was to test the importance of nNOS-derived NO in protection versus CKD progression $[1,5]$ since $7 \mathrm{NI}$ is reported to be nNOS selective. However, as pointed out by Alderton et al. [13], 7NI inhibits both nNOS and inducible NOS, but not eNOS [13], thus exacerbation of $\mathrm{CN}$-induced $\mathrm{CKD}$ with 7NI could reflect loss of nNOS- and/or iNOS-derived NO. It is difficult to predict the impact of iNOS on CKD progression since induction of iNOS is associated with inflammation which could exacerbate the injury process. Given the lack of eNOS inhibitory actions of 7NI [13] this does suggest that NO deficiency due to the nNOS or possibly iNOS, rather than eNOS, play a major role in CKD progression in the WT.

Because there are no eNOS-selective inhibitors, we used the eNOS KO mouse on the C57Bl6 background and in these animals a lifetime of eNOS-dependent NO deficiency does produce significant structural damage to the kidney, as also reported by others $[14,15]$. Although some indices of renal structural damage were moderately increased when eNOS KO mice were subjected to $\mathrm{CN}$, there was little overall amplification of injury. This was unexpected since progression of diabetic nephropathy is greatly exacerbated in the eNOS KO mouse $[14,15]$ and sev-
Table 3. Measurements taken at week 11 of mean BP, plasma $\mathrm{NO}_{2}$ $+\mathrm{NO}_{3}=\mathrm{NOx}$ (factored for plasma creatinine) and 24-hour Ccr

\begin{tabular}{|c|c|c|c|}
\hline Group & $\begin{array}{l}\text { Mean BP } \\
\text { mm Hg }\end{array}$ & $\mathrm{PNOx} / \mathrm{Pcr}$ & Ccr, $\mathrm{ml} / \mathrm{min}$ \\
\hline 1 WT sham & $98 \pm 7$ & $3.64 \pm 1.25$ & $0.335 \pm 0.071$ \\
\hline 2 WT L-NAME & $102 \pm 6$ & $5.20 \pm 0.45$ & $0.656 \pm 0.076^{\mathrm{a}}$ \\
\hline 3 WT 7NI & $89 \pm 3$ & $1.53 \pm 0.31$ & $0.593 \pm 0.080^{\mathrm{a}}$ \\
\hline 4 WT CN & $97 \pm 2$ & $1.66 \pm 0.46$ & $0.193 \pm 0.044^{\mathrm{a}}$ \\
\hline 5 WT CN/L-NAME & $112 \pm 5$ & $0.89 \pm 0.15^{\mathrm{a}}$ & $0.167 \pm 0.033^{b}$ \\
\hline $6 \mathrm{WT} \mathrm{CN/7NI}$ & $87 \pm 7$ & $0.83 \pm 0.26^{\mathrm{a}}$ & $0.328 \pm 0.019^{\mathrm{b}}$ \\
\hline 7 eNOS KO sham & ND & $0.53 \pm 0.07^{\mathrm{a}}$ & $0.234 \pm 0.049$ \\
\hline 8 eNOS KO CN & ND & $0.25 \pm 0.04^{\mathrm{a}}$ & $0.124 \pm 0.019^{c}$ \\
\hline
\end{tabular}

$\mathrm{ND}=$ Not determined. All numbers are given in table 1.

${ }^{\mathrm{a}} \mathrm{p}<0.05$ WT sham vs. WT experimental.

${ }^{\mathrm{b}} \mathrm{p}<0.05$ WT CN vs. WT experimental.

c eNOS sham vs. eNOS CN.

eral groups have reported exacerbation of injury with different models of renal mass reduction CKD in the eNOS KO $[16,17]$. We did, however, note a huge amplification of the already substantial albuminuria when the eNOS KOs were subjected to CN. Presumably, this heralds future amplification of the structural damage, since heavy proteinuria exacerbates CKD progression [18]. Nevertheless, as noted above, the response to $7 \mathrm{NI}$ in the $\mathrm{WT}+\mathrm{CN}$ suggests that NO deficiency due to eNOS is less important in CKD progression in the WT.

In conclusion, we find that chronic NOS inhibition with both L-NAME and 7NI render the resistant C57Bl6 mouse susceptible to renal mass reduction-induced CKD. This is most likely due predominantly to nNOS or iNOS inhibition, based on the 7NI studies. The sham eNOS KO mouse has substantial kidney damage which is only slightly worse at 11 weeks post- $\mathrm{CN}$, although the albuminuria is greatly exacerbated. Overall, these findings support the hypothesis that chronic NOS inhibition promotes CKD progression.

\section{Acknowledgments}

The authors thank Lennie Samsell, Kevin Engels and Harold Snellen for technical assistance. These studies were supported by NIH grant No. R01 DK56843.

\section{Disclosure Statement}

No conflicts of interest to disclose. 


\section{References}

1 Baylis C: Nitric oxide deficiency in chronic kidney disease. Am J Physiol Renal 2008; 294:F1-F9.

-2 Kren S, Hostetter TH: The course of the remnant kidney model in mice. Kidney Int 1999; 56:333-337.

$\checkmark 3$ Ma LJ, Fogo AB: Model of robust induction of glomerulosclerosis in mice: importance of genetic background. Kidney Int 2003;64: 350-355.

4 Erdely A, Wagner L, Muller V, Szabo A, Baylis C: Protection of Wistar-Furth rats from chronic renal disease is associated with maintained renal nitric oxide synthase. J Am Soc Nephrol 2003; 14:2526-2533.

5 Smith C, Merchant M, Fekete A, Nyugen HL, Oh P, Tain YL, Klein JB, Baylis C: Splice variants of neuronal nitric oxide synthase are present in the rat kidney. Nephrol Dial Transplant 2009;24:1422-1428.

$\checkmark 6$ Gagnon RF, Duguid WP: A reproducible model for chronic renal failure in the mouse. Urol Res 1983;11:11-14

7 Kurihara N, Alfie ME, Sigmon DH, Rhaleb NE, Shesely EG, Carretero OA: Role of nNOS in blood pressure regulation in eNOS null mutant mice. Hypertension 1998;32:856861.
8 Clapp W1, Croker BP: Adult kidney; in Mills SE (ed): Histology for Pathologists, ed 3 Philadelphia, Lippincott Williams \& Wilkins, 2007, pp 839-907.

$\checkmark 9$ Erdely A, Freshour G, Smith C, Engels K, Olson J, Baylis C: Protection against puromycin aminonucleoside (PAN)-induced chronic renal disease in the Wistar-Furth. Am J Physiol Renal 2004;287:F81-F89.

10 Erdely A, Freshour G, Tain YL, Engels K, Baylis C: DOCA/NaCl-induced chronic kidney disease: a comparison of renal nitric oxide production in resistant and susceptible rat strains. Am J Physiol Renal 2007; 292:F192-F196.

11 Erdely A, Freshour G, Baylis C: Resistance to renal damage by chronic nitric oxide synthase inhibition in the Wistar-Furth rat. Am J Physiol 2006;290:R66-R72.

12 Boffa JJ, Tharaux PL, Placier S, Ardaillou R, Dussaule JC, Chatziantoniou C: Angiotensin II activates collagen type I gene in the renal vasculature of transgenic mice during inhibition of nitric oxide synthesis: evidence for an endothelin-mediated mechanism. Circulation 1999;100:1901-1908.

13 Alderton WK, Cooper CE, Knowles RG: Nitric oxide synthases: structure, function and inhibition. Biochem J 2001;357:593-615.
14 Kanetsuna Y, Takahashi K, Nagata M, Gannon MA, Breyer MD, Harris RC, Takahashi T: Deficiency of endothelial nitric-oxide synthase confers susceptibility to diabetic nephropathy in nephropathy-resistant inbred mice. Am J Pathol 2007;170:1473-1484.

15 Nakagawa T, Sato W, Glushakova O, Heinig M, Clarke T, Campbell-Thompson M, Yuzawa Y, Atkinson MA, Johnson RJ, Croker B: Diabetic endothelial nitric oxide synthase knockout mice develop advanced diabetic nephropathy. J Am Soc Nephrol 2007;18: 539-550.

16 Yamashita C, Tazawa N, Ohkita M, Matsumura Y: Exaggerated renal pathology of partial ablation induced chronic renal failure in eNOS-deficient mice. Biol Pharm Bull 2008;31:1029-1031.

- 17 Nakayama T, Sato W, Kosugi T, Zhang L, Campbell-Thompson M, Yoshimura A, Croker BP, Johnson RJ, Nakagawa T: Endothelial injury due to eNOS deficiency accelerates the progression of chronic renal disease in the mouse. Am J Physiol Renal Physiol 2009; 296:F317-F327.

18 Abbate M, Zoja C, Remuzzi G: How does proteinuria cause progressive renal damage? J Am Soc Nephrol 2006;17:2974-2984. 